\title{
Combining symbolic computation and theorem proving: some problems of Ramanujan
}

\author{
Edmund Clarke Xudong Zhao \\ January 1994 \\ CMU-CS-94-103
}

\author{
School of Computer Science \\ Carnegie Mellon University \\ Pittsburgh, PA 15213
}

\begin{abstract}
One way of building more powerful theorem provers is to use techniques from symbolic computation. The challenge problems in this paper are taken from Chapter 2 of Ramanujan's Notebooks. They were selected because they are non-trivial and require the use of symbolic computation techniques. We have developed a theorem prover based on the symbolic computation system Mathematica that can prove all the challenge problems completely automatically. The axioms and inference rules for constructing the proofs are also briefly discussed.
\end{abstract}

This research was sponsored in part by the Avionics Laboratory, Wright Research and Development Center, Aeronautical Systems Division (AFSC), U.S. Air Force, Wright-Patterson AFB, Ohio 45433-6543 under Contract F33615-90-C-1465, ARPA Order No. 7597, and in part by National Science Foundation under Contract Number CCR-9217549.

The views and conclusions contained in this document are those of the authors and should not be interpreted as representing the official policies, either expressed or implied, of the U.S. government. 
Keywords: Theorem Prover, Symbolic computation, Mathemmatica, Analytica 


\section{Introduction}

One way of building more powerful theorem provers is to use techniques from symbolic computation. So far, there has been very little research in this direction. The challenge problems in this paper are taken from Chapter 2 of Ramanujan's Notebooks [1]. They were selected because they are non-trivial and require the use of symbolic computation techniques. The preface to Chapter 2 describes the problems as being "fairly elementary", but states that "several of the formulas are very intriguing and evince Ramanujan's ingenuity and cleverness." We suspect that several of the problems would prove quite challenging for many mathematics graduate students even with the help of a symbolic computation system.

We have developed a theorem prover based on the symbolic computation system Mathematica [8] that can prove all the challenge problems completely automatically. This theorem prover uses many of the same techniques that we incorporated in an earlier theorem prover called Analytica [2, 3]. We plan to describe the theorem prover in greater detail in a forthcoming paper.

Although decision procedures like Gosper's algorithm [4] can prove some identities involving summations, we have not found a decision procedure that can handle the problems proposed here. Moreover, decision procedures only give the final result without intermediate steps. Our theorem prover produces readable proofs in which each intermediate step is justified by an axiom or a rule of inference that can be checked by the user. This usually gives greater insight into why the theorem is true.

\section{Axioms that are used in proving the theorems}

In addition to the simplification rules that are provided by the symbolic computation system, the following axioms are also needed for proving the theorems. All of these axioms are simple identities about summations. However, no symbolic computation system, including Mathematica, implements these identities so that they can be applied in both directions. In order to use these axioms effectively, a theorem prover (like the one we have developed) must be constructed so that cycles are avoided and termination is guaranteed.

1. $\sum_{k=i}^{j} f(k)=\sum_{k=1}^{j} f(k)-\sum_{k=1}^{i-1} f(k)$

2. $\sum_{k=1}^{-n} f(k)=-\sum_{k=-n+1}^{0} f(k)$

3. $\sum_{k=1}^{n}(f(k)+g(k))=\sum_{k=1}^{n} f(k)+\sum_{k=1}^{n} g(k)$

4. $\sum_{k=1}^{n} c f(k)=c \sum_{k=1}^{n} f(k)$

5. $\sum_{k=1}^{n+1} f(k)=\sum_{k=1}^{n} f(k)+f(n+1)$

6. $\sum_{k=1}^{2 n}(-1)^{k} f(k)=\sum_{k=1}^{n} f(2 k)-\sum_{k=1}^{n} f(2 k-1)$

7. $\sum_{k=1}^{n a} f(k)=\sum_{j=1}^{n} \sum_{k=0}^{a} f(n k+j)$

8. $\sum_{k=1}^{n} f(k+c)=\sum_{k=c+1}^{n+c} f(k)$ 


\section{List of problems}

The list of challenge problems is given below. All of these problems can be proved automatically by the theorem proving system we have developed. This system uses the rules for summation given in the previous section and is similar to another theorem prover that we have built called Analytica [2,3].

Ramanujan used two abbreviations in stating the theorems. We will use these abbreviations as well.

$$
\begin{gathered}
\Phi(x, n) \equiv 1+2 \sum_{k=1}^{n} \frac{1}{-(k x)+k^{3} x^{3}} \\
\varphi(x, n) \equiv \sum_{k=1}^{n} \frac{1}{-(k x)+k^{3} x^{3}}
\end{gathered}
$$

\subsection{Problems involving summation of rational functions}

1. $\sum_{k=1}^{n} \frac{1}{n+k}=\frac{n}{2 n+1}+\varphi(2, n)$

2. $\sum_{k=1}^{n} \frac{n-k}{n+k}=2 n \varphi(2, n)-\frac{n}{2 n+1}$

3. $\sum_{k=1}^{2 n+1} \frac{1}{n+k}=\Phi(3, n)$

4. $\left(\sum_{k=1}^{n} \frac{1}{n+k}\right)+\left(\sum_{k=0}^{n} \frac{1}{2 n+2 k+1}\right)=\Phi(4, n)$

5. $\left(\sum_{k=1}^{4 n+1} \frac{(-1)^{k+1}}{k}\right)+\frac{1}{2}\left(\sum_{k=1}^{2 n} \frac{(-1)^{k+1}}{k}\right)=\Phi(4, n)$

6. $\Phi(6, n)=\frac{2}{3}\left(\sum_{k=1}^{n} \frac{1}{n+k}\right)+\left(\sum_{k=0}^{2 n} \frac{1}{2 n+2 k+1}\right)$

7. $2 \Phi(4, n)=\Phi(2,2 n)+\frac{\Phi(2, n)}{2}+\frac{1}{(4 n+1)(4 n+2)}$

8. $\Phi(4, n)=\frac{1}{2}\left(\sum_{k=n+1}^{2 n} \frac{1}{k}\right)+\left(\sum_{k=2 n+1}^{4 n+1} \frac{1}{k}\right)$

9. $2 \Phi(6, n)+\frac{\Phi(2, n)}{3}=\Phi(3, n)+\Phi(2,3 n)+\frac{2}{(6 n+1)(6 n+2)(6 n+3)}$

10. $\sum_{k=n+1}^{A_{r}} \frac{1}{k}=r+2\left(\sum_{k=1}^{r}(r-k)\left(\sum_{j=A_{k-1}+1}^{A_{k}} \frac{1}{(3 j)^{3}-3 j}\right)\right)+2 r \varphi\left(3, A_{0}\right)$ where $A_{0}=1, A_{n+1}=3 A_{n}+1$. 


\subsection{Problems involving infinite summations}

Given that $\lim _{n \rightarrow \infty}\left(\sum_{k=1}^{n} \frac{1}{k}-\ln n\right)=\gamma$, where $\gamma$ is the Euler constant, the following identities can also be proved.

1. $\Phi(2, \infty)=2 \ln (2)$

2. $\Phi(3, \infty)=\ln (3)$

3. $\Phi(4, \infty)=\frac{3}{2} \ln (2)$

4. $\Phi(6, \infty)=\frac{\ln (3)}{2}+\frac{\ln (4)}{3}$

5. $\sum_{k=1}^{\infty} \frac{1}{(2(2 k-1))^{3}-2(2 k-1)}=\frac{\ln (2)}{4}$

6. $\sum_{k=1}^{\infty} \frac{2(-1)^{k}}{(2 k)^{3}-2 k}=\ln (2)-1$

7. $\sum_{k=1}^{\infty} \frac{1}{(3(2 k-1))^{3}-3(2 k-1)}=\frac{\ln (3)}{4}-\frac{\ln (2)}{3}$

8. $\sum_{k=1}^{\infty} \frac{2(-1)^{k}}{(3 k)^{3}-3 k}=\frac{4}{3} \ln (2)-1$

\subsection{Problems about the arctan function}

The equations in this section can be proved using the standard trigonometric identities for the arctan function. These identities are not provided by Mathematica and may be treated as axioms.

1. $\sum_{k=1}^{2 n+1} \arctan \left(\frac{1}{n+k}\right)=\frac{\pi}{4}+\left(\sum_{k=1}^{n} \arctan \left(\frac{10 k}{\left(3 k^{2}+2\right)\left(9 k^{2}-1\right)}\right)\right)$

2. $2\left(\sum_{k=1}^{n+1} \arctan \left(\frac{1}{n+k}\right)\right)=\arctan \left(\frac{n+1}{n}\right)+\left(\sum_{k=1}^{n} \arctan \left(\frac{2 k}{8 k^{4}+2 k^{2}+1}\right)\right)+2\left(\sum_{k=1}^{n} \arctan \left(\frac{1}{k\left(4 k^{2}+3\right)}\right)\right)$

3. $\left(\sum_{k=1}^{n} \arctan \left(\frac{1}{n+k}\right)\right)+\left(\sum_{k=0}^{n} \arctan \left(\frac{1}{2 n+2 k+1}\right)\right)$ $=\frac{\pi}{4}+\left(\sum_{k=1}^{n} \arctan \left(\frac{9 k}{32 k^{4}+22 k^{2}-1}\right)\right)+\left(\sum_{k=1}^{n} \arctan \left(\frac{4 k}{128 k^{4}+8 k^{2}+1}\right)\right)$

4. $\sum_{k=0}^{r-1} \arctan \left(\frac{2}{(n+2 k+1)^{2}}\right)=\arctan \left(\frac{2 r}{n^{2}+2 n r+1}\right)$

5. $\sum_{k=0}^{\infty} \arctan \left(\frac{2}{(n+2 k+1)^{2}}\right)=\arctan \left(\frac{1}{n}\right)$

6. $\sum_{k=1}^{\infty} \arctan \left(\frac{2}{(n+k)^{2}}\right)=\arctan \left(\frac{2 n+1}{n^{2}+n-1}\right)$

7. $\left(\sum_{k=1}^{\infty}(-1)^{k+1} \arctan \left(\frac{2}{(n+k)^{2}}\right)=\arctan \left(\frac{1}{n^{2}+n+1}\right)\right.$

8. $\sum_{k=1}^{\infty} \arctan \left(\frac{1}{2(n+k)^{2}}\right)=\arctan \left(\frac{1}{2 n+1}\right)$

9. $\sum_{k=1}^{\infty} \arctan \left(\frac{2}{k^{2}}\right)=\frac{3 \pi}{4}$ 
10. $\sum_{k=1}^{\infty} \arctan \left(\frac{1}{2 k^{2}}\right)=\frac{\pi}{4}$

11. $\sum_{k=1}^{\infty}(-1)^{k+1} \arctan \left(\frac{2}{k^{2}}\right)=\frac{\pi}{4}$

12. $\sum_{k=1}^{\infty} \arctan \left(\frac{1}{(1+\sqrt{2} k)^{2}}\right)=\frac{\pi}{8}$

13. $\sum_{k=1}^{\infty} \arctan \left(\frac{8}{(2 k-1+\sqrt{5})^{2}}\right)=\frac{\pi}{2}$

14. $\sum_{k=0}^{\infty} \arctan \left(\frac{2}{(2 k+1)^{2}}\right)=\frac{\pi}{2}$

\section{A sample proof generated by our system}

In this section, the proof for identity 1 in Subsection 3.3 is presented to illustrate how our prover works. In the proofs of the identities in this and the next sections, some elementary properties of the harmonic numbers $H_{n}=\sum_{k=1}^{n} \frac{1}{k}$ as well as the properties of summations given in Section 2 are used. All of the simplification steps involving summation and the harmonic numbers are implemented directly by our theorem prover. None can be done by Mathematica alone. We have been extremely careful to use only very general rules.

\section{Theorem :}

$$
\left(\sum_{k=1}^{4 n+1} \frac{(-1)^{k+1}}{k}\right)+\frac{1}{2}\left(\sum_{k=1}^{2 n} \frac{(-1)^{k+1}}{k}\right)=\Phi(4, n)
$$

Proof : left hand side :

$$
\left(\sum_{k=1}^{4 n+1} \frac{(-1)^{k+1}}{k}\right)+\frac{1}{2}\left(\sum_{k=1}^{2 n} \frac{(-1)^{k+1}}{k}\right)
$$

simplify

$$
-\frac{1}{2} \sum_{k=1}^{2 n} \frac{(-1)^{k}}{k}-\left(\sum_{k=1}^{1+4 n} \frac{(-1)^{k}}{k}\right)
$$

change summation with alternating sign terms to a sum of two summations

$$
-\frac{1}{4} \sum_{k=1}^{n} \frac{1}{k}-\frac{1}{2} \sum_{k=1}^{2 n} \frac{1}{k}+\frac{1}{2} \sum_{k=1}^{n} \frac{1}{-1+2 k}+\left(\sum_{k=1}^{1+2 n} \frac{1}{-1+2 k}\right)
$$

use harmonic number notation

$$
-\frac{1}{4} H_{n}-\frac{1}{2} H_{2 n}+\frac{1}{2} \sum_{k=1}^{n} \frac{1}{-1+2 k}+\left(\sum_{k=1}^{1+2 n} \frac{1}{-1+2 k}\right)
$$

rewrite summation to standard ranges

$$
\frac{1}{-1+2(1+2 n)}-\frac{1}{4} H_{n}-\frac{1}{2} H_{2 n}+\frac{1}{2} \sum_{k=1}^{n} \frac{1}{-1+2 k}+\left(\sum_{k=1}^{2 n} \frac{1}{-1+2 k}\right)
$$

simplify

$$
\frac{1}{1+4 n}-\frac{1}{4} H_{n}-\frac{1}{2} H_{2 n}+\frac{1}{2} \sum_{k=1}^{n} \frac{1}{-1+2 k}+\left(\sum_{k=1}^{2 n} \frac{1}{-1+2 k}\right)
$$


change index of summations

$$
\frac{1}{1+4 n}-\frac{1}{4} H_{n}-\frac{1}{2} H_{2 n}+\frac{1}{4} \sum_{k=\frac{1}{2}}^{-\frac{1}{2}+n} \frac{1}{k}+\frac{1}{2} \sum_{k=\frac{1}{2}}^{-\frac{1}{2}+2 n} \frac{1}{k}
$$

use harmonic number notation

$$
\frac{1}{1+4 n}+\frac{1}{4} H_{-\frac{1}{2}+n}-\frac{1}{4} H_{n}-\frac{1}{2} H_{2 n}+\frac{1}{2} H_{-\frac{1}{2}+2 n}
$$

simplify harmonic numbers

$$
\frac{1}{1+4 n}-\frac{1}{4} H_{n}-\frac{1}{2} H_{2 n}+\frac{1}{4}\left(-H_{n}+2 H_{2 n}\right)+\frac{1}{2}\left(-H_{2 n}+2 H_{4 n}\right)
$$

simplify

$$
\frac{1}{1+4 n}-\frac{1}{2} H_{n}-\frac{1}{2} H_{2 n}+H_{4 n}
$$

right hand side :

$$
\Phi(4, n)
$$

rewrite rational expressions

$$
1+2\left(-\frac{1}{4} \sum_{k=1}^{n} \frac{1}{k}+\frac{1}{2} \sum_{k=1}^{n} \frac{1}{-1+4 k}+\frac{1}{2} \sum_{k=1}^{n} \frac{1}{1+4 k}\right)
$$

simplify

$$
1-\frac{1}{2} \sum_{k=1}^{n} \frac{1}{k}+\left(\sum_{k=1}^{n} \frac{1}{-1+4 k}\right)+\left(\sum_{k=1}^{n} \frac{1}{1+4 k}\right)
$$

use harmonic number notation

$$
1-\frac{1}{2} H_{n}+\left(\sum_{k=1}^{n} \frac{1}{-1+4 k}\right)+\left(\sum_{k=1}^{n} \frac{1}{1+4 k}\right)
$$

change index of summations

$$
1-\frac{1}{2} H_{n}+\frac{1}{4} \sum_{k=\frac{3}{4}}^{-\frac{1}{4}+n} \frac{1}{k}+\frac{1}{4} \sum_{k=\frac{5}{4}}^{\frac{1}{4}+n} \frac{1}{k}
$$

use harmonic number notation

$$
1+\frac{1}{4} H_{-\frac{1}{4}+n}-\frac{1}{2} H_{n}+\frac{1}{4}\left(-4+H_{\frac{1}{4}+n}\right)
$$

simplify

$$
\frac{1}{4} H_{-\frac{1}{4}+n}-\frac{1}{2} H_{n}+\frac{1}{4} H_{\frac{1}{4}+n}
$$

simplify harmonic numbers

$$
\frac{1}{4} H_{-\frac{1}{4}+n}-\frac{1}{2} H_{n}+\frac{1}{4}\left(\frac{1}{\frac{1}{4}+n}-H_{-\frac{1}{4}+n}-2 H_{2 n}+4 H_{4 n}\right)
$$

simplify

$$
\frac{1}{1+4 n}-\frac{1}{2} H_{n}-\frac{1}{2} H_{2 n}+H_{4 n}
$$

both side are same 


\section{Outline of a more complicated proof}

This section contains part of the proof of identity 10 in Subsection 3.1. The proof has twenty steps, some of which are quite complicated. However, the time required to complete the proof is only about two minutes.

Theorem :

$$
\sum_{k=n+1}^{A_{r}} \frac{1}{k}=r+2\left(\sum_{k=1}^{r}(r-k)\left(\sum_{j=A_{k-1}+1}^{A_{k}} \frac{1}{(3 j)^{3}-3 j}\right)\right)+2 r \varphi\left(3, A_{0}\right)
$$

Proof :

left hand side :

$$
\sum_{k=n+1}^{A_{r}} \frac{1}{k}
$$

use harmonic number notation

$$
-H_{n}+H_{A_{r}}
$$

right hand side :

$$
r+2\left(\sum_{k=1}^{r}(r-k)\left(\sum_{j=A_{k-1}+1}^{A_{k}} \frac{1}{(3 j)^{3}-3 j}\right)\right)+2 r \varphi\left(3, A_{0}\right)
$$

simplify

$$
r+2 r\left(\sum_{k=1}^{n} \frac{1}{-3 k+27 k^{3}}\right)+2 r\left(\sum_{k=1}^{r} \sum_{j=1+A_{-1+k}}^{A_{k}} \frac{1}{-3 j+27 j^{3}}\right)-2\left(\sum_{k=1}^{r} k\left(\sum_{j=1+A_{-1+k}}^{A_{k}} \frac{1}{-3 j+27 j^{3}}\right)\right)
$$

rewrite rational expressions

$$
\begin{aligned}
& r+2 r\left(-\frac{1}{3} \sum_{k=1}^{n} \frac{1}{k}+\frac{1}{2} \sum_{k=1}^{n} \frac{1}{-1+3 k}+\frac{1}{2} \sum_{k=1}^{n} \frac{1}{1+3 k}\right) \\
& +2 r\left(-\frac{1}{3} \sum_{k=1}^{r} \sum_{j=1+A_{-1+k}}^{A_{k}} \frac{1}{j}+\frac{1}{2} \sum_{k=1}^{r} \sum_{j=1+A_{-1+k}}^{A_{k}} \frac{1}{-1+3 j}+\frac{1}{2} \sum_{k=1}^{r} \sum_{j=1+A_{-1+k}}^{A_{k}} \frac{1}{1+3 j}\right) \\
& -2\left(-\frac{1}{3} \sum_{k=1}^{r} k\left(\sum_{j=1+A_{-1+k}}^{A_{k}} \frac{1}{j}\right)+\frac{1}{2} \sum_{k=1}^{r} k\left(\sum_{j=1+A_{-1+k}}^{A_{k}} \frac{1}{-1+3 j}\right)+\frac{1}{2} \sum_{k=1}^{r} k\left(\sum_{j=1+A_{-1+k}}^{A_{k}} \frac{1}{1+3 j}\right)\right)
\end{aligned}
$$

-. ( 7 steps $)$

$$
\begin{aligned}
& r-\frac{2}{3} r H_{n}+\left(\sum_{j=1}^{n} \frac{1}{-1+3 j}\right)-\left(\sum_{j=1}^{A_{r}} \frac{1}{-1+3 j}\right)-r\left(\sum_{j=1}^{A_{r}} \frac{1}{-1+3 j}\right)+\left(\sum_{j=1}^{n} \frac{1}{1+3 j}\right) \\
& -\left(\sum_{j=1}^{A_{r}} \frac{1}{1+3 j}\right)-r\left(\sum_{j=1}^{A_{r}} \frac{1}{1+3 j}\right)+r\left(\sum_{k=1}^{n} \frac{1}{-1+3 k}\right)+r\left(\sum_{k=1}^{n} \frac{1}{1+3 k}\right)-\frac{2}{3} r\left(\sum_{k=1}^{r} H_{A_{k}}\right) \\
& -\frac{2}{3}\left(H_{n}-H_{A_{r}}+\left(\sum_{k=1}^{r} H_{A_{k}}\right)\right)+\frac{2}{3} r\left(H_{n}-H_{A_{r}}+\left(\sum_{k=1}^{r} H_{A_{k}}\right)\right)+\frac{2}{3} \sum_{k=1}^{r} k H_{A_{k}}
\end{aligned}
$$




$$
\begin{aligned}
& -\frac{2}{3}\left(-\left(r H_{A_{r}}\right)+\left(\sum_{k=1}^{r} k H_{A_{k}}\right)\right)+\left(\sum_{k=1}^{r} \sum_{j=1}^{A_{k}} \frac{1}{-1+3 j}\right)+r\left(\sum_{k=1}^{r} \sum_{j=1}^{A_{k}} \frac{1}{-1+3 j}\right) \\
& -\left(r\left(\left(\sum_{j=1}^{n} \frac{1}{-1+3 j}\right)-\left(\sum_{j=1}^{A_{r}} \frac{1}{-1+3 j}\right)+\left(\sum_{k=1}^{r} \sum_{j=1}^{A_{k}} \frac{1}{-1+3 j}\right)\right)+\left(\sum_{k=1}^{r} \sum_{j=1}^{A_{k}} \frac{1}{1+3 j}\right)\right. \\
& +r\left(\sum_{k=1}^{r} \sum_{j=1}^{A_{k}} \frac{1}{1+3 j}\right)-r\left(\left(\sum_{j=1}^{n} \frac{1}{1+3 j}\right)-\left(\sum_{j=1}^{A_{r}} \frac{1}{1+3 j}\right)+\left(\sum_{k=1}^{r} \sum_{j=1}^{A_{k}} \frac{1}{1+3 j}\right)\right) \\
& (6 \text { stpes }) \\
& \frac{1}{1+3 n}-H_{n}+H_{3 n}+H_{A_{r}}-H_{A_{1+r}}-\left(\sum_{k=1}^{r} H_{A_{k}}\right)+\left(\sum_{k=1}^{r} H_{A_{1+k}}\right)
\end{aligned}
$$

increase or decrease indices

$$
\frac{1}{1+3 n}-H_{n}+H_{3 n}+H_{A_{r}}-H_{A_{1+r}}-\left(\sum_{k=1}^{r} H_{A_{k}}\right)+\left(\sum_{k=2}^{1+r} H_{A_{k}}\right)
$$

simplify range of summation

$$
\frac{1}{1+3 n}-H_{n}+H_{3 n}-H_{1+3 n}+H_{A_{r}}
$$

use harmonic number notation

$$
-H_{n}+H_{A_{r}}
$$

both sides are the same

\section{Related Work}

There has been relatively little work on using symbolic computation techniques in automatic theorem proving besides our own research on Analytica [2, 3]. Suppes and Takahashi [6] have combined a resolution theorem prover with the Reduce system, but their prover is only able to check very small steps and does not appear to have been able to handle very complicated proofs. London and Musser [5] have also experimented with the use of Reduce for program verification, but did not consider theorems from other areas of mathematics or computer science. Bundy [7] has investigated the use of induction for finding closed forms for summations. He is able to handle some very complicated examples. However, his techniques are not applicable to the summations in this paper since they do not have closed forms.

\section{References}

[1] B.C.Berndt, Ramanujan's Notebooks, Part I, Springer-Verlag, 1985, pp 25-43.

[2] E.M.Clarke, X.Zhao, Analytica - An Experiment in Combining Theorem Proving and Symbolic Computation, Technical Report, School of Computer Science, Carnegie Mellon University, CMU-CS-92-147, Oct. 1992. 
[3] E.M.Clarke, X.Zhao, Analytica - A theorem prover for Mathematica, The Mathematica Journal, Vol. 3, Issue 1, 1993, pp 56-71.

[4] R.W.Gosper, Indefinite Hypergeometric sums in MACSYMA, Proc. MACSYMA Users Conference, Berkeley CA, 1977, pp 237-252.

[5] R.L.London and D.R.Musser, The Application of a Symbolic Mathematical System to Program Verification, Technique Report, USC Information Science Institute.

[6] P.Suppes and S.Takahashi, An Interactive Calculus Theorem-prover for Continuity Properties, Journal of Symbolic Computation, No.7, 1989, pp 573-590.

[7] T.Walsh, A.Nunes, and A.Bundy, The Use of Proof Plans to Sum Series, Proc. of 11th International Conference on Automated Deduction, June 1992.

[8] S. Wolfram. Mathematica: A System for Doing Mathematics by Computer, Wolfram Research Inc., 1988. 